\title{
Editorial
}

\section{Poor Medication Adherence in Women-The Tip of a Gender Inequity Iceberg?}

\author{
Pragna Rao, MD, $\mathrm{PhD}^{1}$ \\ ${ }^{1}$ Advisor, Manipal Global Education Services, Bengaluru, Karnataka, \\ India
}

Ind J Car Dis Wom:2020;5:313-314

The Global Burden of Disease warns that among women across the world, including India, stroke and ischemic heart disease are the major causes of death. Yet, at a tertiary care hospital in Hyderabad, India, approximately $61 \%$ of the women patients were found to have low-to-medium level of medication adherence for the secondary prevention of cardiovascular disease (CVDs). This is the finding of a study entitled "Medication adherence and its determinants among women of cardiovascular disease of tertiary care hospital, Hyderabad," which was published in this journal. The authors felt that there was a paucity of studies, specifically among women, to understand the interplay of various determinants influencing medication adherence. Hence, this hospital-based study was taken up, which used the Morisky medication adherence scale (MMAS-4) to estimate the level of medication adherence. A considerable percentage (90\%) of the women included in the study possessed educational qualifications which were less than a college degree, and over $90 \%$ were unemployed or poorly skilled. Economically, $57 \%$ of the women belonged to the upper and upper middle class. Among this population, prevalence of medication adherence among women afflicted with CVDs using MMAS-4 was found to be high among $39 \%$, medium in $51 \%$, and low in $10 \%$ of the study sample. The reasons given for unsatisfactory compliance were forgetfulness (11\%), poverty $(9.1 \%)$, irregular supply of drugs (18\%), and limited supply among 7.4\% of the women patients. Socioeconomic determinants such as poverty, unemployment, illiteracy, limited supply of drugs, and high-cost of medicines were the significant reasons for low-medication adherence. Patient-related determinants such as the unwillingness of patients to take medicines, especially for a long duration, forgetfulness, and the reluctance to continue medications once the symptoms subsided were also significant factors. Of major concern was the fact that irregular supply of drugs at the government center became a significant reason for nonadherence. The study concludes that better medication adherence needs to be addressed

Address for correspondence

Pragna Rao, MD, PhD, Advisor, Manipal Global Education Services, Manipal Towers, Old HAL Airport Road, Bengaluru, Karnataka 560008, India (e-mail: drpragnarao@gmail.com). by targeting the common determinants such as forgetfulness which can be reduced by putting reminders in mobile phones, creating health education about the importance of intake of drugs, thereby preventing complications with regular follow-up, and physician's taking up the responsibility to effectively communicate the lifelong need for medications in addition to healthy lifestyle modifications

Although the reasons for nonadherence are multifactorial, studies conducted among the Indian population (including both males and females) have blamed the patients' lack of awareness about the disease and its complications. The second commonly cited reason for nonadherence is "forgetfulness." To extend the same simplistic arguments among women patients would be committing both social and medical injustice. An earlier study by Gupta et al examined the socioeconomic barriers for low adherence to antihypertensive medication among women in India. Poor access to healthcare, out-of-pocket payments, borrowing money, lack of insurance, and cost of medicines were cited as major deterrents to medication adherence. ${ }^{1}$

The problem may lie even deeper than that. Among women in South Asia, gender-based discrimination can cause adverse health outcomes. Beginning from food allocation, and being unable to access healthcare services and finances to pay for their treatment, women face odds in society. Saikia et $\mathrm{al}^{2}$ argue that in poor families, health-related expenditure usually leads to compromising the health needs of female family members. Even in families where finance is not a concern, women have less power to demand that their needs be taken care of, due to lack of income-earning means, lack of support from the community, and social norms and perceptions. Thus, the gender differential in healthcare expenditure in India is perhaps the result of powerful synergies between socioeconomic status and a male-dominated society.

Medication nonadherence could be a silent, unspoken result of these factors.

Any kind of gender discrimination is a cause of concern, irrespective of the disease. An economic discussion

(c) 2020. Women in Cardiology and Related Sciences.

This is an open access article published by Thieme under the terms of the Creative Commons Attribution-NonDerivative-NonCommercial-License, permitting copying and reproduction so long as the original work is given appropriate credit. Contents may not be used for commercial purposes, or adapted, remixed, transformed or built upon. (https://creativecommons.org/licenses/by-nc-nd/4.0/).

Thieme Medical and Scientific Publishers Pvt. Ltd. A-12, 2nd Floor, Sector 2, Noida-201301 UP, India 
paper written by authors of the Indian Statistical Institute, New Delhi, ${ }^{3}$ indicated that gender discrimination accounts for $73 \%$ of the gender difference in cumulative total expenditure. Of this, $64 \%$ of the difference is on account of gender discrimination in cumulative medical expenditure. When such is the case, medication nonadherence is a natural fallout. Most of the differences emerge from expenditures before the patient even reaches the tertiary care hospital.

Medication nonadherence will increase inequalities in health outcomes between the genders, and also impose a larger economic impact by way of healthy life years lost to disease. Besides, children in families, particularly nuclear families, get affected when a female caregiver, like a mother, is not healthy.

This calls for systemic societal and governmental action to introduce gender-inclusive health education in India, emphasizing the significance of NCDs and the treatment associated with them. However, the larger need remains, which involves empowering women by engaging them in economic activities to reduce gender-based discrimination in healthcare.

Possible gender-based problems can be addressed by more innovative, informed, and gender-sensitive planning, including targeted education and culturally sensitive communication campaigns. Teleconsultations with women healthcare providers might play an important role in boosting medication adherence.

Better medication adherence, therefore, goes beyond just obeying the doctor's prescription. Its ramifications are both social and economic, and they play a vital role in supporting a healthy society.

\section{Conflict of Interest}

None declared.

\section{References}

1 Gupta S, Dhamija JP, Mohan I, Gupta R. qualitative study of barriers to adherence to antihypertensive medication among rural women in India. Int J Hypertens 2019;2019:5749648

2 Saikia N, Moradhvaj, Bora JK. Gender difference in health-care expenditure: evidence from India Human Development Survey. PLoS One 2016;11(7):e0158332

3 Batra A, Gupta I, Mukhopadhyay A. Does discrimination drive gender differences in health expenditure on adults: evidence from Cancer patients in rural India. Indian Statistical Institute Discussion Paper. 2014:14-03 